\title{
Pobreza y subdesarrollo rural en Colombia. Análisis desde la Teoría del Sesgo Urbano
}

\author{
Laura Vanessa López Muñoz (Colombia)*
}

\section{Resumen}

La pobreza en las zonas rurales ha sido una condición persistente a lo largo de la historia colombiana. Con el propósito de profundizar el entendimiento de las dinámicas que favorecen la perdurabilidad de esta situación, en este artículo se realiza un análisis del subdesarrollo rural en Colombia desde la perspectiva que ofrece la Teoría del Sesgo Urbano, propuesta por Michael Lipton en 1977. A partir de una revisión histórica, se ha encontrado que el detrimento de las zonas rurales del país se explica en gran medida por los procesos políticos y económicos que han sido impulsados por las élites urbanas para su beneficio y el desarrollo de las ciudades. Los resultados muestran bajos niveles en la provisión de servicios públicos para las poblaciones rurales, en comparación con los altos niveles registrados en las áreas urbanas. Se presenta, además, una interpretación del conflicto armado colombiano y de los flujos migratorios del campo a la ciudad como consecuencias agregadas y perpetuadores del sesgo urbano en Colombia.

\section{Palabras clave}

Economía Política; Sesgo Urbano; Pobreza; Subdesarrollo; Colombia.

Fecha de recepción: agosto de 2018 • Fecha de aprobación: noviembre de 2018

\section{Cómo citar este artículo}

López Muñoz, Laura Vanessa. (2019). Pobreza y subdesarrollo rural en Colombia. Análisis desde la Teoría del Sesgo Urbano. Estudios Políticos (Universidad de Antioquia), 54, pp. 59-81. http://doi.org/10.17533/udea.espo.n54a04

\footnotetext{
* Profesional en Relaciones Internacionales. Magíster en Gobernanza y Desarrollo. Docente del Departamento de Ciencia Política y Relaciones Internacionales, Universidad del Norte, Colombia. Correo electrónico: vlopezl@uninorte.edu.co Orcid: https://orcid.org/0000-0002-7605-625X
} 


\title{
Poverty and Rural Underdevelopment in Colombia. An Analysis from the Urban Bias Theory
}

\begin{abstract}
Poverty in rural areas has been a persistent condition throughout Colombian history. In order to broadening the understanding of those dynamics that contribute to the perpetuation of poverty, this article analyzes Colombian rural underdevelopment from the perspective of the Urban Bias Theory, proposed by Michael Lipton in 1977, and based on an historical review. The study found that the detriment of the rural regions of the country is due to the political and economic processes promoted by urban elites, who seek for their own benefit and urban development. The results show low provision of public services for the rural inhabitants, when compared to urban areas. This paper also presents an interpretation of the Colombian armed conflict and migration flows from the countryside to the cities as additional consequences and perpetuators of the urban bias in Colombia.
\end{abstract}

\section{Keywords}

Political Economy; Urban Bias; Poverty; Underdevelopment; Colombia. 


\section{Introducción}

El subdesarrollo rural en Colombia puede ser entendido como el resultado de la suma de diversos sesgos. Por un lado, se ha encontrado un sesgo de clases en la distribución de recursos para el campo a lo largo de la historia colombiana. Albert Berry (2017, p. 175) afirma que este sesgo ha beneficiado a los grandes terratenientes, quienes han contado con apoyo en forma de créditos o mejora de infraestructura para la explotación de la tierra, mientras que las pequeñas familias agricultoras no han experimentado un apoyo representativo que genere mejoras en su producción y condiciones de vida.

Del mismo modo, se identifica la existencia de un sesgo de género. Teresa Castaño (2015, pp. 5-10) señala que en la economía nacional no se reconocen las actividades realizadas por las mujeres rurales. A pesar de que estas mujeres se dedican al trabajo del hogar, a la agricultura familiar y a la construcción cuando es necesario, ninguna de estas actividades es remunerada. No obstante, sin negar la existencia de estos sesgos en el contexto colombiano, este trabajo se enfocará en el análisis de la pobreza rural desde la perspectiva del sesgo urbano.

Michael Lipton (1977) sostenía que el progreso económico de los países en vía de desarrollo estaba siendo guiado por un sesgo urbano, es decir, las políticas de estas naciones estaban siendo promovidas por grupos políticos y económicos concentrados en las urbes, que asignaban los recursos para favorecer las áreas urbanas en detrimento de las zonas rurales. Aunque estos argumentos tienen más de cuarenta años, en el caso colombiano la predominancia urbana sobre las áreas rurales demuestra la permanencia de preferencias por los modelos urbanos. Primero, se conoce que las grandes ciudades del país concentran $77 \%$ de la población nacional (World Bank, 2018). Segundo, para 2014 se reportó que mientras 5,1\% de la población urbana vivía en extrema pobreza, $18 \%$ de la población rural vivía en esta condición (DNP, 2015, p. 15). Tal situación es el resultado de una conjunción de políticas que han sido elaboradas por personas en las ciudades y que han dejado un legado de sesgo urbano que permanece en el país.

En este artículo se argumenta, fundamentalmente, que el subdesarrollo rural del país se explica, en gran medida, por una tradición de sesgo urbano. Se realiza una interpretación del contexto histórico y de los factores que han desencadenado en la asignación desigual de recursos en el territorio 
colombiano. Es conocido que las medidas empíricas para analizar el sesgo urbano incluyen la diferencia rural-urbana en la provisión de servicios públicos, la supervaloración de la tasa real de cambio y las tasas netas de impuestos (Pierskalla, 2016, p. 287). En este sentido, para efectos de este trabajo la medición se concentrará en las diferencias en la provisión de servicios públicos en las áreas rurales y urbanas en Colombia.

El artículo hace una revisión de la literatura de la Teoría del Sesgo Urbano y expone, en líneas generales, los determinantes políticos y económicos, así como las manifestaciones del sesgo urbano. Luego, con el objetivo de examinar la aplicación de la teoría de Lipton al caso de estudio que concierne a esta investigación, se identifican los elementos políticos y económicos del sesgo urbano en la historia de Colombia, y se revisa la diferencia rural-urbana en la provisión de servicios públicos. Adicionalmente, se analiza la forma en que el conflicto armado y la migración del campo a la ciudad se han convertido en consecuencias y perpetuadores de la tradición del sesgo urbano en el país. El artículo finaliza con una conclusión que incluye comentarios y recomendaciones con miras a la reducción del sesgo urbano en Colombia.

\section{Revisitando la idea del sesgo urbano}

La Teoría del Sesgo Urbano (Lipton, 1977) ha orientado el análisis de la pobreza hacia asuntos como la prevalencia de un estado mental centrado en la vida y el desarrollo urbano, los giros de precios en contra de la producción del campo y la desigualdad entre las zonas urbanas y rurales. Esto con el fin de proveer luces para el entendimiento de las políticas que han efectuado una ineficiente e injusta dirección de recursos. Se parte de la idea de que la equidad y la eficiencia pueden ser promovidas a través de los procesos políticos (Williams, Duncan, Landell y Unsworth, 2011, p. 529); sin embargo, al ser mal estructurados, estos procesos políticos terminan siendo causantes de la ausencia de crecimiento de una nación.

Cedric Pugh (1996, p. 1049) define el sesgo urbano como una coalición de intereses urbanos que se han organizado exitosamente para influir en la formulación de políticas, generando políticas económicas que crean un desarrollo económico desbalanceado y polarizado en detrimento de los escenarios rurales. El sesgo urbano está representado en el conjunto de medios a través de los cuales los recursos son empleados para favorecer las áreas urbanas (Disney, 1979, p. 167), dificultando así el desarrollo del mundo rural. 
Al analizar el contexto histórico, a pesar de que las instituciones extractivas feudales y los regímenes coloniales imprimieron un legado de sesgos estructurales en contra de la transformación agrícola en los países en vía de desarrollo, han sido necesarios otros elementos para crear una imagen completa del sesgo urbano en la historia contemporánea de estas naciones (Bezemer y Headey, 2008, pp. 1353-1354).

En este sentido, la contemporaneidad trajo consigo una combinación de estrategias proteccionistas y de sustitución de importaciones que fueron promovidas en las décadas de 1960 y 1970 con el propósito de modernizar e industrializar el mundo subdesarrollado. Posteriormente, en la década de 1980 el Banco Mundial y los hacedores políticos incentivaron el establecimiento de políticas económicas para la transformación estructural que no fueron precisamente beneficiosas para el desarrollo del campo. Este conjunto de decisiones políticas y económicas evidenciaron un sesgo urbano determinante para la evolución desigual del campo y la ciudad.

Uno de los principales críticos de la teoría del sesgo urbano ha sido Cristóbal Kay (2009), quien sostiene que un error contundente de esta teoría ha sido ignorar las potenciales sinergias que se pueden encontrar entre agricultura e industria para impulsar el desarrollo rural: «los analistas del SU [sesgo urbano] pierden de vista el dinamismo de los clusters industriales y de las ciudades, así como las importantes contribuciones que la industria puede hacer a la agricultura mediante la provisión de maquinaria, equipos e insumos modernos» (Kay, 2009, p. 14).

En esta afirmación se desconoce que la tesis del sesgo urbano busca enfocar la dinámica política y económica en la que la industria crea riqueza que beneficia a las ciudades y su población, en tanto que las contribuciones de inversión e innovación en las zonas rurales son ineficientes y desiguales. Es relevante mencionar que la idea del sesgo urbano no rechaza la creación de estas sinergias, pero sí señala la gran inversión que se realiza para el desarrollo de los sectores urbanos, mientras que las familias rurales no son receptoras de una inversión similar. Una sinergia e interacción entre sectores agrícola e industrial no es posible sin antes eliminar el sesgo en contra del campo, el cual ha promovido más políticas asistenciales y sociales que de inversión tecnológica e innovación para el desarrollo económico de las familias rurales. Por lo tanto, la noción del sesgo urbano trae consigo ciertos aspectos políticos y económicos que resultan relevantes para el estudio de las estrategias utilizadas en los países en vía de desarrollo (Pierskalla, 2011, p. 32), así como para la explicación de características del subdesarrollo rural. 


\subsection{Los elementos políticos y económicos del sesgo urbano}

El sesgo urbano puede ser visto como una conjunción de condiciones sociopolíticas (Pugh, 1996, p. 1047). La Teoría del Sesgo Urbano reconoce la primacía de la política en los procesos de desarrollo (Lipton, 1977, p. 63): cómo los gobiernos son parte y tienden a apoyar los intereses urbanos en países pobres. Este supuesto se encuentra alineado con la visión de la literatura de economía política que posiciona la política en el centro de los estudios de desarrollo, enfocando la atención en la primacía de la política y del carácter de los Estados (Leftwich, 2000, p. 18).

Por un lado, la idea del sesgo urbano señala la exagerada influencia política de los residentes urbanos (Pierskalla, 2011, p. 2). La pobreza perdura en los países pobres debido a que estos son desarrollados por y para individuos que gobiernan, dirigen recursos, incentivos, educación e investigaciones desde y para la ciudad (Lipton, 1977, p. 68). Además, aun cuando los recursos son destinados a la agricultura sirven para perpetuar a la élite urbana, por ejemplo, cuando los terratenientes y la clase industrial se benefician de los subsidios para la agricultura (Disney, 1979, p. 167). Para algunos autores la distinción de personas rurales y urbanas que establece Lipton (1977) es radical dado que, por ejemplo, llama urbanos a grupos de terratenientes. Sin embargo, afirmaciones como «los terratenientes muchas veces invirtieron en empresas urbanas, y los capitalistas urbanos adquirieron tierras y propiedades rurales, generalmente por prestigio social y por razones políticas, facilitando así la formación de alianzas entre ellos» (Kay, 2009, p. 10), ponen en evidencia la naturaleza urbana y poderosa de aquellas personas que concentran grandes cantidades de tierra y tienen la capacidad de influencia en la toma de decisiones políticas. Al final, resulta complicado sostener que un terrateniente que maneja o invierte en empresas urbanas es una persona rural.

Por otro lado, las decisiones y medidas económicas tomadas por los gobiernos han sido contundentes para la creación de la brecha rural-urbana contemporánea. En las décadas de 1950 y 1960 las políticas de modernización en los países en vía de desarrollo estuvieron enfocadas en la sustitución de importaciones y estrategias proteccionistas para estimular la industrialización (Martin, 2003, pp. 187-190). La sustitución de importaciones consistió en el establecimiento de facilidades de producción doméstica para bienes de manufactura a partir de grandes inversiones hacia el sector industrial (Baer, 1972, p. 95). 
Las políticas proteccionistas llevaron a que los costos del campo aumentaran, pues algunos de los bienes que se introducían a la producción rural tenían precios mayores a los que pudieron haber tenido si hubieran estado expuestos a los mercados y la competencia extranjera (Pugh, 1996, p. 1047). Esta sobreevaluación del campo afectó negativamente el potencial de las exportaciones agrícolas debido a que los campesinos tenían que vender sus productos en injustas condiciones de mercado (Jones y Corbridge, 2010, p. 10).

El giro de precios, es decir, la forma en la cual los precios se giraron en contra del sector rural, al generar un sobreprecio de los aportes al sector rural y una reducción del precio de la producción rural, evidenció y profundizó el sesgo urbano por ser una medida en favor del proceso de industrialización (Anochiwa y Enyoghasim, 2016, p. 8; Lipton, 1977, p. 308).

Luego, en las décadas de 1980 y 1990, muchos países en vía de desarrollo adoptaron un paquete de medidas económicas institucionales conocidas como préstamos de ajuste estructural, que incluyeron la desregulación y privatización económica (Leftwich, 2000, p. 12). A cambio de préstamos del Banco Mundial y del Fondo Monetario Internacional se intentó promover la liberalización de los mercados. A pesar de que estas medidas buscaban el ajuste de precios y el crecimiento económico, los resultados no fueron beneficiosos en general, teniendo en cuenta que los principales veinte receptores de préstamos no alcanzaron un crecimiento razonable, sino que mostraron fuertes distorsiones macroeconómicas (Easterly, 2005, p. 7).

Consecuentemente, la globalización, las remesas, la introducción de plantas agroprocesadoras y supermercados en el campo, la diversificación de las fuentes de ingresos a través de las artesanías, actividades de construcción, turismo, entre otras, constituyen factores que han generado cambios y relaciones que dispersan la línea divisoria entre el campo y la ciudad (Kay, 2009, pp. 19-21). No obstante, sería bueno reconocer que estas transformaciones e interacciones han sucedido en gran medida por la dificultad de obtener ganancias a partir de las actividades tradicionales del campo y no necesariamente porque la agricultura esté generando excedentes.

En este sentido, la persistencia de políticas tomadas desde las ciudades, que han apoyado ciertos programas y sectores sobre otros, ha generado efectos negativos como el aumento de la desigualdad dentro de los países desde finales del siglo xx (Jones y Corbridge, 2010, p. 16). 


\subsection{Manifestación del sesgo urbano en las sociedades en vía de desarrollo}

Distintos autores han reconocido que la pobreza rural está fuertemente determinada por la acción del gobierno en la creación de políticas sesgadas hacia lo urbano, que resultan en la poca inversión en las áreas rurales (Anochiwa y Enyoghasim, 2016; Jones y Corbridge, 2010; Lipton, 1977; Pugh, 1996). Se debe tener cuidado al pensar que la teoría del sesgo urbano crea una mera división espacial, como lo ha afirmado Kay (2009, p. 9), cuando en realidad esta teoría analiza distinciones económicas y sociales entre las esferas rural y urbana, teniendo incluso como una de sus medidas la diferencia en provisión de servicios públicos entre el campo y la ciudad.

Las élites urbanas en los países en vía de desarrollo, que con su poder económico y político han logrado estructurar el desarrollo hacia la ciudad, han influido en la creación de la disparidad de bienes públicos (Majumdar, Mani y Mukand, 2004, p. 138). Esta disparidad está demostrada en, por ejemplo, las diferencias en el acceso a agua potable entre poblaciones rurales y urbanas (Bezemer y Headey, 2008, p. 12). En el ámbito mundial existe una gran desigualdad rural-urbana en el porcentaje de la población que utiliza servicios

[ 66 ] de agua potable tratada de forma segura. De acuerdo con los datos provistos por el World Bank (2018), en 2015 el 85,12\% de la población urbana tenía acceso a este tipo de agua, mientras que solo $54,81 \%$ de la población rural disfrutaba de ella. Esta es una tendencia clara en distintas regiones del mundo (véase tabla 1).

Tabla 1. Población rural y urbana que utiliza servicios de agua potable tratada de forma segura.

\begin{tabular}{|l|c|c|}
\hline \multirow{2}{*}{ País } & \multicolumn{2}{|c|}{ Población que utiliza servicios de agua potable tratada de forma segura } \\
\cline { 2 - 3 } & Rural & Urbana \\
\hline Camboya & $16 \%$ & $55 \%$ \\
\hline Etiopia & $3,93 \%$ & $38 \%$ \\
\hline Marruecos & $39 \%$ & $89 \%$ \\
\hline Nicaragua & $29,55 \%$ & $79,16 \%$ \\
\hline Perú & $19,92 \%$ & $58,38 \%$ \\
\hline
\end{tabular}

Fuente: elaboración propia a partir de World Bank (2018).

Otra evidencia de la desigualdad en la asignación de recursos entre el campo y la ciudad en los países en vía de desarrollo es la disparidad en la 
cobertura de educación. La Unesco (2005), reporta que los residentes rurales tienen bajos niveles de alfabetismo comparados con los residentes urbanos. Por ejemplo, en 2004 la tasa de alfabetismo estuvo estimada en $72 \%$ en las áreas urbanas de Pakistán, mientras que en las áreas rurales esta tasa era de 44\%. Asimismo, Etiopía presentó una tasa de alfabetismo de $74 \%$ en áreas urbanas, comparado con una tasa de $23 \%$ en áreas rurales (p. 171).

Los efectos de esta desigualdad rural-urbana en la provisión de educación han afectado la creación de capital humano en los países en vía de desarrollo (Jones y Corbridge, 2010, p. 10), demostrando que los gobiernos tienen un efecto directo sobre los determinantes inmediatos del crecimiento económico: el capital, la tecnología y la eficiencia (Weil, 2005, p. 341).

Por otro lado, Pugh (1996, p. 1047) afirma que un efecto del sesgo urbano es la migración del campo a la ciudad, la cual es estimulada por la posibilidad de obtener mejores ingresos y oportunidades de trabajo en escenarios urbanos. Así también, Sumon Majumdar, Anandi Mani y Sharun Mukand (2004, pp. 154-155) afirman que un sesgo en la provisión de bienes públicos tiene repercusiones para la decisión de migrar. En este sentido, de acuerdo con el World Bank (2018), durante el periodo de 1960-2017 la población urbana del mundo ha crecido de 1 billón a 4.1 billones, mientras que la población rural ha presentado un crecimiento más lento: de 2 billones a 3.4 billones en el mismo periodo.

Por lo tanto, para realizar el análisis del sesgo urbano en un Estado resulta necesario revisar la información concerniente a su historia política y económica. Asimismo, para conocer la persistencia de este sesgo es primordial examinar los datos sobre la provisión de servicios públicos y los flujos migratorios internos del campo a la ciudad. Este es el propósito de la segunda y tercera parte del presente artículo, que busca dilucidar el asunto del sesgo urbano en Colombia a partir de una operacionalización básica pero pragmática de la teoría propuesta por Lipton.

\section{Consolidación del sesgo urbano en Colombia}

Desde el periodo de la colonia española los modelos de extracción y trabajo de la tierra creados por la Corona generaron conflictos sociales y políticos en las distintas regiones. Desde el siglo XVIII se empezaron a formar los latifundios en Colombia, sin embargo, es en las décadas siguientes a la Independencia, con la redistribución del poder político y las riquezas, que 
se presentó la excesiva concentración de la tierra (Mörner y Herrera-Salazar, 1974, p. 752), la cual ha desencadenado luchas por una repartición más justa hasta el día de hoy.

Poco a poco, distintas estrategias y alianzas fueron garantizando el dominio de la élite sobre el campo, y fueron consolidando lo que es hoy una larga tradición de sesgo urbano en el Estado colombiano (OECD, 2014, p. 23). Un fuerte vínculo entre los terratenientes y los comerciantes se forjó en la década de 1860, cuando las élites acordaron el desarrollo de las exportaciones agrícolas, estimulando el compromiso con la producción y comercialización de los cultivos para exportación (Reinhardt, 1986, p. 89). Especialmente, en la segunda mitad del siglo xIx, los comerciantes de origen urbano - de Manizales, Medellín y Bogotá- empezaron a constituir el sector exportador de café, convirtiéndose en hacendados, terratenientes, accionistas y, poco a poco, en empresarios industriales (Bejarano, 1980, pp. 124-126).

Luego, durante el periodo de posguerra, el declive del mercado internacional ejerció presión en Colombia, donde se comenzó a implementar la estrategia de sustitución de importaciones para el desarrollo económico y la industrialización, a partir del establecimiento de altas tarifas comerciales [ 68 ] que permitieron un rápido crecimiento de la industria manufacturera (Garay, 1998, p. 456). El campo se vio afectado, pues la clase política idealizaba un país industrializado y no enfocado solo en la agricultura (León, 2004, p. 183).

Años más adelante, en la década de 1980, el Gobierno inició un proceso de apertura comercial, introduciendo estrategias de liberalización de importaciones y promoción de exportaciones, e induciendo un cambio tecnológico hacia el trabajo calificado (Ocampo, Sánchez, Hernández y Prada, 2004, p. 20). Con la apertura al comercio exterior la situación del campo colombiano se agravó, la agricultura fue sometida a ciertas exigencias en productividad e inversión para poder hacer frente a la competencia exterior, lo que generó en la década de 1990 una reducción de las áreas de cultivos transables - como cereales y algodón-y el aumento del desempleo rural (García, 2002, p. 225).

También hay que considerar que en Colombia las élites políticas han defendido los intereses de la élite económica. Los dos partidos tradicionales colombianos, el Partido Liberal y el Partido Conservador, han representado a la élite del país y han sido protagonistas en los procesos políticos de asignación de recursos. Liberales y conservadores han amparado a la misma élite, aunque 
han diferido en las políticas que han considerado mejores para favorecerla (Reinhardt, 1986, pp. 78-79).

El desenlace del desacuerdo y deseo de poder de los dos partidos tradicionales resultó en un periodo conocido como La Violencia entre 1948 y 1958. Este periodo significó una competencia entre liberales y conservadores por el poder político a través de medios violentos, especialmente en zonas rurales que, sumado a la omisión de las demandas de los campesinos (Kay y Salazar, 2001, p. 173), generó un detrimento de estas regiones, grandes desplazamientos y una concentración mayor de la tierra. Los dos partidos competían por la legitimidad del poder con el propósito de utilizar las posiciones burocráticas y los servicios públicos para recompenzar las lealtades a su partido, manteniendo y fortaleciendo así las relaciones clientelares (Schmidt, 1974, p. 103). La política fue usada como una pantalla para preservar intereses económicos, por ejemplo, lograr la concentración de mayores cantidades de tierra a través de la usurpación a miembros del partido opositor; sin embargo, estas luchas se daban entre los campesinos, pues la élite conformada por los terratenientes, personas de negocios y políticos se resguardaba en la seguridad de las ciudades (Bushnell, 1993).

Luego, en 1958, con la firma del pacto conocido como Frente Nacional, los dos partidos políticos tradicionales establecieron un sistema de rotación del gobierno cada cuatro años, con el cual garantizaron por dieciséis años la protección del poder de las élites. Aunque en la actualidad existe mayor pluralismo político, los partidos poderosos y representantes de la élite del país siguen desempeñando un rol preponderante desde los distintos órganos del Gobierno para la defensa y protección de los intereses industriales y urbanos.

Por otra parte, la ausencia de límites generales para la cantidad de hectáreas que un individuo o entidad puede poseer en Colombia, sumado al desigual acceso a la tierra, ha constituido otro determinante para la acumulación del poder de las élites. En efecto, 80\% de las tierras en el país se encuentran bajo la tenencia de 14\% de los terratenientes (Oxfam, 2013, p. 7), con el agravante de que cerca de $35 \%$ de ese territorio podría estar vinculado al negocio de las drogas (Garay, 2008, p. 17).

Michael Albertus y Oliver Kaplan (2013, p. 199) reconocen que las reformas agrarias son políticamente difíciles de implementar, pues amenazan el statu quo de las élites del país. Por ejemplo, en 1936 se creó la Ley de Tierras, con la cual el gobierno buscaba disminuir los conflictos entre latifundistas y 
campesinos, quienes exigían la abolición de las formas de trabajo opresivas y la adquisición de derechos de propiedad; sin embargo, los resultados de esta ley no fueron satisfactorios, pues los terratenientes reaccionaron expulsando a los campesinos arrendatarios de las tierras y el gobierno no tomó acciones como respuesta (Kay y Salazar, 2001, p. 173). Así, el conjunto de campesinos desposeídos, resultado de las formas de dominación de los terratenientes, fueron conformando los primeros vestigios del proletariado industrial en las ciudades (León, 2004, p. 184).

Además, el sesgo urbano que tienen las políticas económicas en Colombia ha esbozado un paisaje en el cual gran parte de las tierras se encuentra bajo la posesión privada (Maldonado y Martínez, 2016, p. 612). Actualmente, el modelo de desarrollo económico del país está basado en una extracción privada a gran escala de los recursos naturales que permite la explotación de la tierra por compañías agroindustriales y de minería, muchas veces sin la consulta de habitantes locales y cultivadores (Chavarro y Tyrou, 2016). Aunque Kay (2009, p. 14) encuentra problemática la poca consideración que se le da a las economías de escala en la Teoría del Sesgo Urbano, es necesario resaltar que la evidencia de Colombia y otros países destacan el papel más exitoso y eficiente que puede desarrollar la agricultura familiar en la economía rural y nacional (Berry, 2017, p. 82).

Por otro lado, una serie de protestas agrarias se han presentado en la última década en contra de los acuerdos de libre comercio con Estados Unidos y la Unión Europea. Los pequeños campesinos y habitantes de áreas rurales perciben el crecimiento económico de la nación como algo que no los beneficia, sosteniendo que ellos no tienen la capacidad de emparejarse con los precios a los que se encuentran los productos agrícolas en el libre mercado y que el aumento de los costos de los ingresos a la producción agrícola, como el combustible y los fertilizantes, han convertido al cultivo de baja escala en un negocio de pérdidas (OECD, 2014, p. 81).

Incluso en la actualidad en Colombia es evidente el sesgo urbano. Cuando los recursos son dirigidos al sector agrícola están garantizados a compañías y familias urbanas poderosas. La explotación de los recursos naturales está monopolizada por personas urbanas (Maldonado y Martínez, 2016, p. 612). Un ejemplo reciente de esto fue el caso del programa Agro Ingreso Seguro, cuyos negativos resultados se pueden adjudicar, en parte, a la dirección de los recursos nacionales disponibles para el beneficio de unas 
cuantas familias poderosas, frustrando las iniciativas hacia el desarrollo de las áreas rurales. Javier Mejía-Cubillos (2012, pp. 16-22) sostiene que el confuso criterio establecido para la distribución de los recursos de este programa benefició a las empresas agrícolas a que impulsaran su competitividad y productividad, mientras que los pequeños productores seguían encontrando dificultades por los altos costos de producción.

Se ha presentado, hasta este punto, la forma en que los procesos políticos y económicos a lo largo de la historia colombiana han asegurado el poder de las élites urbanas en detrimento de las personas del campo. Como agravante, la toma de decisiones políticas respecto al desarrollo rural se ha visto caracterizada por un enfoque social en vez de económico (OECD, 2014, p. 23), representando también un asunto para revisión de los esfuerzos que está teniendo el Gobierno hacia el fortalecimiento y desarrollo de estas regiones desde la inversión en capitales traducidos en bienes y servicios.

\section{Efectos del sesgo urbano en Colombia}

El nivel de desigualdad en Colombia es uno de los más altos del mundo. La gran diferencia en las condiciones de vida rural-urbana ejemplifica las profundas tensiones sociales del país (Parra, Ordóñez y Acosta, 2013, p. 16). Este estudio no niega la existencia de altos índices de pobreza urbana, ni la desigualdad en las áreas urbanas reflejada, por ejemplo, en un coeficiente Gini urbano de 0.54 para 2010 (DNP y DANE, 2012, p. 63). Sin embargo, la falta de oportunidades para los habitantes rurales y la brecha de pobreza rural-urbana pone en evidencia una deuda histórica de Colombia con los territorios rurales (Martínez, Pertuz y Ramírez, 2016).

En este mismo orden, los indicadores socioeconómicos para los hogares rurales en Colombia son más bajos que aquellos de los hogares urbanos (OECD, 2014, p. 75). A pesar de que la pobreza rural y el índice de recuento de la pobreza en las zonas rurales han decrecido, en la tabla 2 se muestra que las áreas urbanas han tenido índices de pobreza más bajos que los de las áreas rurales, lo que demuestra que el desarrollo de Colombia ha sido más evidente en las ciudades.

Se mencionó previamente que una de las medidas empíricas del sesgo urbano es la revisión de las diferencias en provisión de servicios públicos (Pierskalla, 2016, p. 287). De esta forma, los niveles de cobertura de servicios 
públicos fueron significativamente diferentes en las zonas urbanas y rurales del país en 2010 (Profamilia, 2011). Los datos exponen desigualdades en la provisión de servicios de electricidad, agua potable y sistemas de alcantarillado entre los hogares rurales y urbanos (véase tabla 3).

Tabla 2. Brecha de pobreza e índice de recuento de pobreza en las zonas rurales y urbanas.

\begin{tabular}{|l|c|c|c|}
\hline & Año & Rural & Urbana \\
\hline Brecha de pobreza a nivel nacional & $\mathbf{2 0 1 0}$ & $21,6 \%$ & $13,1 \%$ \\
\cline { 2 - 4 } & $\mathbf{2 0 1 5}$ & $15,7 \%$ & $8,7 \%$ \\
\hline Índice de recuento de pobreza & $\mathbf{2 0 0 2}$ & $61,7 \%$ & $45,5 \%$ \\
\cline { 2 - 4 } nacional (población) & $\mathbf{2 0 1 5}$ & $40,3 \%$ & $24,1 \%$ \\
\hline
\end{tabular}

Fuente: elaboración propia a partir de World Bank (2018).

Tabla 3. Niveles de provisión de servicios públicos en áreas rurales y urbanas de Colombia.

\begin{tabular}{|l|c|c|}
\hline \multicolumn{1}{|c|}{ Servicio público } & Áreas rurales & Áreas urbanas \\
\hline Electricidad & $91 \%$ & $99 \%$ \\
\hline Agua potable & $17 \%$ & $91 \%$ \\
\hline Sistema de alcantarillado & $22 \%$ & $92 \%$ \\
\hline
\end{tabular}

Fuente: elaboración propia a partir de Profamilia (2011).

Además, mientras la población en zonas rurales cuenta con promedio de años de educación de 5.1, en las zonas urbanas es de 8.4, poniendo en evidencia un desinterés por completar los ciclos académicos en las zonas rurales causado por la ausencia de establecimientos educativos próximos, por los altos niveles de pobreza y por el poco conocimiento de cómo la educación puede generar mejores posibilidades (Ministerio de Educación Nacional, 2017). De igual forma, en 2013 la población rural analfabeta mayor de quince años era de $12,5 \%$, mientras que la urbana con estas características era de 3,8\% (DANE, 2014). Así, la ineficiente inversión del gobierno en educación en las zonas rurales genera afectaciones directas al capital humano (Weil, 2005, p. 341), el cual impiden el desarrollo de estas regiones. 
Las políticas implementadas por el Gobierno colombiano para la provisión de servicios públicos han sido claves para el subdesarrollo rural. A través de los procesos políticos y el establecimiento de las reglas del juego se han ido moldeando los resultados económicos y sociales (Weil, 2005, pp. 346-347; Williams et al., 2011, p. 529), afectando los capitales y el desarrollo económico de las zonas rurales. Bien señala Kay (2009, p. 11) que una de las formas de probar las ideas del sesgo urbano es cuestionarse sobre qué podría pasar si este sesgo se elimina o se reduce. Ciertamente, inversiones en servicios públicos como la educación y saneamiento de las personas rurales generarían efectos directos en el capital humano, uno de los determinantes fundamentales del crecimiento económico.

La tradición de sesgo urbano representa un desafío para el desarrollo de Colombia. Las desigualdades en inversión y provisión de servicios han llevado, entre otros, a que los jóvenes rurales perciban al campo colombiano como un escenario que no brinda las oportunidades necesarias para su movilidad social (Martínez, Pertuz y Ramírez, 2016). En este sentido, si el sesgo urbano se redujera se revisarían y propondrían políticas encaminadas al mejoramiento de las zonas rurales a partir de mayores inversiones, aumento de incentivos y mejora de la calidad de vida.

\section{Conflicto armado y migración: consecuencias y perpetuadores del sesgo urbano}

El conflicto armado en Colombia puede ser visto tanto como un resultado o un perpetuador del sesgo urbano. Por un lado, el origen de los grupos armados al margen de la ley puede ser adjudicado a los actos de exclusión de las élites políticas y económicas en contra de distintos sectores de la sociedad colombiana en las dinámicas de desarrollo que llevaron a grupos de individuos a sublevarse en contra de estas prácticas injustas y en respuesta a la poca presencia del Estado en las regiones. Por otro lado, a pesar de que el crimen y la violencia han sido parte de la formación del Estado colombiano (Grajales, 2011, p. 771), los grupos armados ilegales han contribuido a la permanencia de la estructura violenta y del esquema político que ha mantenido a las regiones rurales subdesarrolladas.

Durante el periodo de La Violencia, la represión del gobierno del Partido Conservador contra el movimiento campesino influyó en la transformación de estos en movimientos guerrilleros cooptados por el Partido Liberal, el partido 
de oposición, lo cual condujo al Partido Conservador a organizar sus propias bandas armadas (Kay y Salazar, 2001, p. 173).

A pesar de que el fin de La Violencia culminó con la guerra entre partidos, también significó un punto de quiebre histórico para el país. Grupos revolucionarios adoptaron una nueva visión de nación que buscaba la redistribución del poder y la eliminación del orden tradicional establecido (Sánchez, 2000, pp. 39-40). Sin embargo, el propósito inicial de lucha fue poco a poco reemplazado por aquel de dominio territorial; así, con los recursos económicos que adquirieron fueron consolidando su control en gran parte del territorio nacional (Rangel, 1999, p. 30). Distintas estrategias como asesinatos, secuestros, torturas y reclutamientos forzados fueron utilizadas por los grupos guerrilleros para consolidar su presencia en casi la mitad del territorio colombiano (De La Asuncion, 2000, p. 448).

Unidades de autodefensa se han confrontado con estos grupos guerrilleros durante décadas, y a su vez han generado actos atroces de violencia como desapariciones, masacres y robos, creando así un conflicto complejo y múltiple en el que las élites políticas y económicas del país se han comportado como testigos tolerantes y, en otras ocasiones, como poder [ 74 ] clandestino detrás de estas fuerzas violentas (Sánchez, 2000, pp. 43-44). Es el grado de libertad que las instancias del poder público le han dado a los grupos paramilitares, ya sea por acción u omisión, lo que ha facilitado la capacidad de estos grupos para aumentar su poder y hacer presencia en distintas regiones del país y esferas del Estado (Ramírez, 2007, p. 33).

Por otro lado, el narcotráfico encontró espacio en esta ausencia estatal en distintas regiones del país. El narcotráfico ha desempeñado un papel protagónico en el conflicto colombiano, con un gran escalamiento de sus actuaciones violentas en la década de 1980 y con una evolución del negocio estrechamente ligada con los grupos paramilitares y guerrilleros hasta la actualidad. El narcotráfico volvió a consolidar una estructura latifundista en el país, con el surgimiento de los nuevos terratenientes que han logrado concentrar tierras para el cultivo de coca, sumarse a la élite tradicional e introducirse en las estructuras de la sociedad colombiana y en los procesos de toma de decisiones (Pampillón y Verna, 1995, pp. 183-184).

A lo largo del tiempo, los diferentes actores ilegales del conflicto, sean guerrillas, paramilitares o grupos narcotraficantes, se han convertido en un gran inconveniente para el desarrollo del campo. Las regiones rurales son 
percibidas como inseguras, elevando así la incertidumbre para los inversionistas, disminuyendo el valor de la tierra y reduciendo las oportunidades económicas para las poblaciones que habitan en estas zonas (Ibáñez y Moya, 2010, p. 145). Además, la violencia ha tenido efectos negativos en el capital cultural y social, al generar, por ejemplo, la suspensión de actividades escolares, desplazamientos forzados (Perfetti, 2005, p. 166) y, por ende, una afectación a todas las prácticas cotidianas.

A lo mencionado por Majumdar, Mani y Mukand (2004, pp. 154-155) respecto a los efectos del sesgo en la provisión de bienes públicos sobre la decisión de migrar, se le suma el conflicto como factor impulsador de estas migraciones en Colombia. El conflicto ha sido la principal causa de los desplazamientos del campo a la ciudad. Desde que inició el conflicto armado en Colombia, cerca de 5.7 millones de personas habían sido desplazadas de sus hogares en el campo (IFAD, 2016). Esta diáspora rural a las ciudades ha sido soportada por la percepción de las áreas rurales como lugares peligrosos (OECD, 2014, p. 62).

Es así como este conflicto, por medio de las migraciones de la población campesina a las ciudades, ha contribuido al acelerado proceso de urbanización que reflejó un crecimiento de más de treinta millones en la población urbana entre 1960 y 2017 (World Bank, 2018). En las décadas de 1950 y 1960 hubo un aumento de la demanda de mano de obra y del mejoramiento de la infraestructura y provisión de servicios en las zonas urbanas debido a los flujos migratorios ocasionados por la violencia y la industrialización que estaba experimentando Colombia; luego, desde la década de 1970 se han empezado a desarrollar distintas iniciativas urbanas como la densificación de los grandes centros económicos del país —Bogotá, Medellín, Cali y Barranquilla-, para acomodar a su población de una forma más eficiente y sustentable (Álvarez, Parés y Aide, 2013, pp. 36-37). El conflicto y el acelerado proceso de urbanización en Colombia ha sido impulsado por el sesgo urbano; sin embargo, la intensificación del conflicto armado logró reforzar estos procesos de urbanización y, por lo tanto, la concentración de miradas y acciones hacia el desarrollo de las ciudades.

\section{Conclusión}

Los argumentos que Michael Lipton defendió hace más de cuatro décadas con respecto a la existencia de un sesgo urbano que hacía perdurar a las personas pobres en su condición de pobreza (Lipton, 1977) encuentran 
validez en el caso de Colombia. La historia colombiana ilustra cómo los recursos en las zonas rurales no han sido distribuidos de acuerdo al criterio de eficiencia y equidad, lo cual ha generado beneficios desproporcionados para las élites poderosas que, además, son esencialmente urbanas.

La historia contemporánea de Colombia ha estado marcada por la concentración del poder político en las manos de las élites urbanas, así como por la injusta distribución de tierras y políticas económicas proteccionistas seguidas por estrategias de liberalización. Este conjunto de elementos ha sido principalmente determinado por los procesos políticos que se han presenciado en el país, los cuales han sido cruciales para la perpetuación de las desigualdades entre las regiones urbanas y rurales. Así, las brechas de pobreza entre las ciudades y el campo se ven reflejadas en la gran diferencia en la provisión de bienes y servicios públicos.

De igual forma, el conflicto armado y la diáspora forzada a las ciudades han servido como indicadores del abandono del campo por parte del Gobierno. La gran presencia de fuerzas ilegales en las diferentes zonas rurales fue creando una percepción de estas como áreas peligrosas, lo cual no solo fue determinante para la reducción de las inversiones, sino también para

[ 76 ] la generación de migraciones forzadas hacia aquellos espacios entendidos como seguros.

El propósito de este artículo fue evidenciar cómo el sesgo urbano se ha consolidado y ha persistido como un problema estructural en Colombia. La sociedad colombiana funciona con un estado mental (Lipton, 1977, p. 63) centrado en la vida y el desarrollo urbano; por esto, el abandono de la población rural se ha convertido en una tradición en Colombia. En este sentido, la deficiente capacidad de desarrollo de las zonas rurales en el país está determinado por las políticas que se ejecutan, que en este caso evidencian un sesgo urbano.

El canal principal a través del cual se puede reducir la desigualdad ruralurbana en Colombia es por medio de la mitigación de la brecha de inversión entre el campo y la ciudad (Berdegué et al., 2015, p. 67). La sociedad colombiana está desperdiciando una gran proporción de su capital físico, humano y ambiental disponible para generar riqueza colectiva (Garay, 2008, p. 16). Además, el surgimiento de un campesinado de clase media podría aparecer si los tomadores de decisiones políticas incorporaran en sus agendas 
estrategias enfocadas en la redistribución de tierras y al desarrollo rural (Parra, Ordóñez y Acosta, 2013, p. 18).

Teniendo en cuenta que el poder de las élites en Colombia es tanto económico como político, un enfoque crítico puede desempeñar un papel clave en este asunto. Lipton (1977, p. 332) afirma que con el fin de reducir el sesgo urbano los políticos, estudiantes y pensadores deben involucrarse con las comunidades rurales, analizando junto con estas poblaciones aquellos factores que perpetúan sus condiciones de pobreza. Esta afirmación de Lipton se puede considerar apropiada y vigente para los tiempos actuales en el escenario colombiano. A través de la aplicación de perspectivas críticas, se puede fortalecer el interés de las personas por participar en espacios de gobernanza y desarrollo local.

Partiendo de los argumentos de Amartya Sen (1994) sobre la educación como creadora de capacidades y contribuidora a la mejora de condiciones de vida, la expansión del conocimiento y la educación puede dar origen a reflexiones críticas por parte de las poblaciones rurales. Esto porque el aumento de oportunidades, de educación en este caso, aumenta las capacidades para participar en el desarrollo económico y social (Pugh, 1996, p. 1052). En todo caso, los niveles actuales de educación dejan cierto espacio para desarrollar estrategias que promuevan el aumento de consciencia política y de los niveles de participación.

Finalmente, se debe dirigir mayor atención al entendimiento de la subyacente política económica en las políticas rurales (Pierskalla, 2016, p. 304). Si bien el sesgo urbano no alcanza a explicar completamente el subdesarrollo rural en el contexto nacional, pues han existido otros factores que han influido en la asignación de recursos como el sesgo de clases, de género y el conflicto armado, esta teoría resulta apropiada para el entendimiento de la situación histórica de la pobreza rural en Colombia y para analizar los elementos que fomentan las inversiones desiguales en el campo y la ciudad.

\section{Referencias bibliográficas}

1. Albertus, Michael \& Kaplan, Oliver. (2013). Land Reform as a Counterinsurgency Policy: Evidence from Colombia. Journal of Conflict Resolution, 57 (2), pp. 198-231. https://doi.org/10.1177/0022002712446130

2. Álvarez-Berríos, Nora L.; Parés-Ramos, Isabel K. \& Aide, T. Mitchell. (2013). Contrasting Patterns of Urban Expansion in Colombia, Ecuador, Peru, and Bolivia 
between 1992 and 2009. Ambio, 42 (1), pp. 29-40. https://doi.org/10.1007/s13280012-0344-8

3. Anochiwa, L. I. \& Enyoghasim, Michael. (2016). Urban Bias, Urban Crises, and Development in Nigeria: Controversies and Contending Issues. EPRA International Journal of Economic and Business Review, 4 (6), pp. 5-11.

4. Baer, Werner. (1972). Import substitution and industrialization in Latin America: experiences and interpretations. Latin American Research Review, 7 (1), pp. 95-122.

5. Bejarano, Jesús Antonio. (1980). Los estudios sobre la historia del café en Colombia. Cuadernos de Economía, 1 (2), pp. 115-140.

6. Berdegué, Julio A.; Carriazo, Fernando; Jara, Benjamin; Modrego, Félix y Soloaga, Isidro. (2015). Cities, Territories, and Inclusive Growth: Unraveling UrbanRural Linkages in Chile, Colombia, and Mexico. World Development, 73, pp. 56-71. https://doi.org/10.1016/j.worlddev.2014.12.013

7. Berry, Albert. (2017). Avance y fracaso en el agro colombiano, siglos xx y XXI. Bogotá, D. C.: Universidad del Rosario.

8. Bezemer, Dirk \& Headey, Derek. (2008). Agriculture, Development, and Urban Bias. World Development, 36 (8), pp. 1342-1364. https://doi.org/10.1016/j. worlddev.2007.07.001

9. Bushnell, David. (1993). The Making of Modern Colombia: A Nation in Spite of Itself. Berkeley, Los Angeles \& London: University of California.

10. Castaño, Teresa. (2015). La mujer rural y la agricultura familiar en Colombia:

[ 78 ] detrás de la economía del cuidado está una mujer que le aporta al país. Bogotá, D. C.: Minsalud, FAO.

11. Chavarro, Juan Sebastián y Tyrou, Emma. (2016). Renewed Social Outcry in Colombia: La Minga, the Struggle of the Marginalized. Council on Hemispheric Affairs. Retrieved from http://www.coha.org/renewed-social-outcry-in-colombia-laminga-the-struggle-of-the-marginalized/

12. De La Asuncion, Alexandra. (2000). Colombia: The Ignored Humanitarian Crisis. The University of Miami Inter-American Law Review, 31 (3), pp. 439-462.

13. Departamento Administrativo Nacional de Estadística (DANE). (2014). Encuesta nacional de calidad de vida 2013. Recuperado de https://bit.ly/2BT4zq7

14. Departamento Nacional de Planeación (DNP). (2015). Diagnóstico de la pobreza rural. Colombia 2002-2014. Recuperado de https://bit.ly/2qis05k

15. Disney, Richard. (1979). Why Poor People Stay Poor: Urban Bias in World Development by Michael Lipton; The Vast Majority: A Journey to the World's Poor by Michael Harrington. The Journal of Modern African Studies, 17 (1), pp. 167-168. https://doi.org/10.1017/S0022278X00005334

16. Departamento Nacional de Planeación (DNP) y Departamento Administrativo Nacional de Estadística (DANE). (2012). Pobreza monetaria en Colombia: nueva metodología y cifras 2002-2010. Resultados segunda fase de la Mesep. Recuperado de https://www.dane.gov.co/files/noticias/Pobreza_nuevametodologia.pdf 
17. Easterly, William. (2005). What did Structural Adjustment Adjust?: The Association of Policies and Growth with Repeated IMF and World Bank Adjustment Loans. Journal of Development Economics, 76 (1), pp. 1-22. https://doi.org/10.1016/ S0304-3878(04)00087-2

18. Garay, Luis Jorge (dir.). (1998). Colombia: estructura industrial e internacionalización 1967-1996. Bogotá, D. C.: DNP. Recuperado de https://bit. ly/2QCOoW7

19. Garay, Luis Jorge. (2008). En torno a la economía política de la exclusión social en Colombia. Revista de Economía Institucional, 5 (8), pp. 15-31.

20. García, José Guillermo. (2002). Liberalización, cambio estructural y crecimiento económico en Colombia. Cuadernos de Economía, 21 (36), pp. 189-244.

21. Grajales, Jacobo. (2011). The Rifle and the Title: Paramilitary Violence, Land Grab and Land Control in Colombia. Journal of Peasant Studies, 38 (4), pp. 771-792. https://doi.org/10.1080/03066150.2011.607701

22. Ibáñez, Ana María \& Moya, Andrés. (2010). Do Conflicts Create Poverty Traps? Asset Losses and Recovery for Displaced Households in Colombia. In: Di Tella, Rafael, Edwards, Sebastian \& Schargrodsky, Ernesto (eds.). The Economics of Crime: Lessons for and from Latin America (pp. 137-172). Chicago: University of Chicago.

23. International Fund for Agricultural Development (IFAD). (2016). Investing in Rural People in Colombia. Roma: International Fund for Agricultural Development. Retrieved from https://bit.ly/2E4K9fs

24. Jones, Gareth A. \& Corbridge, Stuart. (2010). The Continuing Debate about Urban Bias: The Thesis, its Critics, its Influence and its Implications for PovertyReduction Strategies. Progress in Development Studies, 10 (1), pp. 1-18. https://doi. org/10.1177/146499340901000101

25. Kay, Cristóbal y Salazar, Graciela. (2001). Estructura agraria, conflicto y violencia en la sociedad rural de América Latina. Revista Mexicana de Sociología, 63 (4), pp. 159-195. https://doi.org/10.2307/3541472

26. Kay, Cristóbal. (2009). Reflexiones sobre desarrollo rural y estrategias de desarrollo: exploración de sinergias, erradicación de pobreza. Debate Agrario, 2009 (44), pp. 1-29.

27. Leftwich, Adrian. (2000). States of Development: On the Primacy of Politics in Development. Cambridge: Polity.

28. León Palacios, Paulo Cesar. (2004). Literature on Colombian Industrialisation: Balance and Perspectives. Innovar, 14 (23), pp. 182-207.

29. Lipton, Michael. (1977). Why Poor People Stay Poor: A Study of Urban Bias in World Development. London: Maurice Temple Smith.

30. Majumdar, Sumon; Mani, Anandi \& Mukand, Sharun W. (2004). Politics, Information and the Urban Bias. Journal of Development Economics, 75 (1), pp. 137165. https://doi.org/10.1016/j.jdeveco.2003.08.003 
31. Maldonado, Juan Mayr \& Martínez, Luisz Olmedo. (2016). Indigenous Peoples, Natural Resources, and Peacebuilding in Colombia. In: Bruch, Carl; Muffett, Carroll \& Nichols, Sandra S. (eds.). Governance, Natural Resources, and Post-Conflict Peacebuilding (pp. 605-625). London: Earthscan. https://doi. org/10.4324/9780203109793-29

32. Martin, Will. (2003). Developing Countries' Changing Participation in World Trade. The World Bank Research Observer, 18 (2), pp. 187-203. https://doi. org/10.1093/wbro/lkg008

33. Martínez-Restrepo, Susana; Pertuz, María Cecilia y Ramírez, Juan Mauricio. (2016). La situación de la educación rural en Colombia, los desafíos del posconflicto y la transformación del campo. Recuperado de https://bit.ly/2AU0WP2

34. Mejía-Cubillos, Javier. (2012). Agro, Ingreso Seguro en perspectiva: Un análisis de políticas públicas. MPRA Paper, 39998. Recuperado de https://bit.ly/2QAu3AB

35. Ministerio de Educación Nacional. (2017). Plan especial de educación rural. Hacia el desarrollo rural y la construcción de paz. Recuperado de http://www. congresoeducacionruralcoreducar.com/images/Doc_web/10.-PEER_06-2017.pdf

36. Mörner, Magnus y Herrera-Salazar, Ricardo. (1974). La hacienda hispanoamericana en la historia: un esquema de reciente investigación y debate. Desarrollo Económico, 13 (52), pp. 741-773. https://doi.org/10.2307/3466291

37. Ocampo, José Antonio; Sánchez, Fabio José; Hernández, Gustavo Adolfo y Prada, María Fernanda. (2004). Crecimiento de las exportaciones y sus efectos sobre [ 80 ] el empleo, la desigualdad y la pobreza en Colombia. Documento Cede, 2004-3. Recuperado de https://bit.ly/2xPLfru

38. Organisation for Economic Co-operation and Development (OECD). (2014). OECDTerritorialReviews:Colombia2014.https://doi.org/10.1787/9789264224551-en

39. Oxfam. (2013). Divide and Purchase: How Land Ownership is Being Concentrated in Colombia. Retrieved from https://bit.ly/2KWW3cx

40. Pampillón, Rafael y Verna, Gérard. (1995). El narcotráfico en Colombia. Política Exterior, 9 (45), pp. 179-192.

41. Parra-Peña, Rafael I.; Ordóñez, Liliana A. y Acosta, Camilo A. (2013). Pobreza, brechas y ruralidad en Colombia. Coyuntura Económica, 43 (1), pp. 15-36.

42. Perfetti, Mauricio. (2005). Estudio sobre la educación para la población rural en Colombia. Recuperado de http://red-ler.org/estudio_educacion_poblacion_rural_ colombia.pdf

43. Pierskalla, Jan H. (2011). Urban Bias and Democracy: The Causal Effect of Elections on Rural Public Goods Provision. APSA 2011 Annual Meeting Paper.

44. Pierskalla, Jan H. (2016). The Politics of Urban Bias: Rural Threats and the Dual Dilemma of Political Survival. Studies in Comparative International Development, 51 (3), pp. 286-307. https://doi.org/10.1007/s12116-015-9194-2

45. Profamilia. (2011). Encuesta Nacional de Demografía y salud 2010. Recuperado de https://bit.ly/2jXdnjm 
46. Pugh, Cedric. (1996). Urban Bias, the Political Economy of Development and Urban Policies for Developing Countries. Urban Studies, 33 (7), pp.1045-1060. https://doi.org/10.1080/00420989650011492

47. Ramírez, Gloria Inés. (2007). Colombia: paramilitarismo y Estado. Política Exterior, 21 (118), pp. 32-38.

48. Rangel, Alfredo. (1999). Colombia: la guerra irregular en el fin de siglo. América Latina Hoy, 23, pp. 29-36.

49. Reinhardt, Nola. (1986). The Consolidation of the Import-Export Economy in Nineteenth-Century Colombia A Political-Economic Analysis. Latin American Perspectives, 13 (1), pp. 75-98. https://doi.org/10.1177/0094582X8601300104

50. Sánchez, Gonzalo. (2000). War and Politics in Colombian Society. International Journal of Politics, Culture, and Society, 14 (1), pp. 19-49. https://doi. org/10.1023/A:1007804828973

51. Sen, Amartya. (1994). Beyond Liberalization: Social Opportunity and Human Capability. New Delhi: Institute of Social Sciences.

52. Schmidt, Steffen W. (1974). La Violencia Revisited: The Clientelist Bases of Political Violence in Colombia. Journal of Latin American Studies, 6 (1), pp. 97-111. https://doi.org/10.1017/S0022216X00008312

53. United Nations Educational, Scientific and Cultural Organization (UNESCO). (2005). Education for All: Global Monitoring Report 2006. Paris: UNESCO. Retrieved from https://bit.ly/2RGNzZP

54. Weil, David N. (2005). Economic Growth. Boston: Addison Wesley Longman.

55. Williams, Gareth; Duncan, Alex; Landell-Mills, Pierre y Unsworth, Sue. (2011). Politics and Growth. Development Policy Review, 29 (1), pp. 529-555. https:// doi.org/10.1111/j.1467-7679.2011.00519.x

56. World Bank. (2018). World Bank Open Data. Retrieved from https://data. worldbank.org 